\title{
Identifying a correlated spin fluctuation in an entangled spin chain subject to a quantum phase transition
}

\author{
Kaoru Shimizu ${ }^{1, *}$ and Yasuhiro Tokura ${ }^{1,2}$ \\ ${ }^{1}$ NTT Basic Research Laboratories, NTT Corporation, 3-1 Morinosato-Wakamiya, Atsugi, Kanagawa 243-0198, Japan \\ ${ }^{2}$ Graduate School of Pure and Applied Sciences, University of Tsukuba, 1-1-1 Tennoudai, Tsukuba, Ibaraki 305-0006, Japan
}

(Received 31 July 2015; revised manuscript received 16 October 2015; published 28 December 2015)

\begin{abstract}
This paper presents a theoretical framework for analyzing the quantum fluctuation properties of a quantum spin chain subject to a quantum phase transition. We can quantify the fluctuation properties by examining the correlation between the fluctuations of two neighboring spins subject to the quantum uncertainty. To do this, we first compute the reduced density matrix $\rho$ of the spin pair from the ground state $|\Psi\rangle$ of a spin chain, and then identify the quantum correlation part $\rho_{q}$ embedded in $\rho$. If the spin chain is translationally symmetric and characterized by a nearest-neighbor two-body spin interaction, we can determine uniquely the form of $\rho_{q}$ as $W|\Phi\rangle\langle\Phi|$ with the weight $W \leqslant 1$, and quantify the fluctuation properties using the two-spin entangled state $|\Phi\rangle$. We demonstrate the framework for a transverse-field quantum Ising spin chain and indicate its validity for more general spin chain models.
\end{abstract}

DOI: 10.1103/PhysRevE.92.062143

PACS number(s): 05.30.Rt, 75.10.Jm, 03.67.Mn, 75.10.Pq

\section{INTRODUCTION}

The dynamics of a quantum phase transition (QPT) in a quantum spin chain is frequently described by the competitive interplay of different types of quantum fluctuation such as antiferromagnetic and ferromagnetic fluctuations. These fluctuations characterize the relative spin orientation of two neighboring spins, which optimizes the energy cost of the spin pair. Although this description helps us greatly to understand the dynamics intuitively, a method for the quantitative identification of the fluctuation has not been established and the description remains qualitative. If the description can be improved and made quantitative, we can obtain an alternative tool for analyzing a low-dimensional quantum spin system. Recent studies of entanglement theories have provided new insights in this direction, where the entangled state of many spins in a system can be analyzed in terms of various entanglement measures [1]. In particular, simple pairwise spin entanglement measures for two spins [2,3] appear to achieve the quantification of the quantum fluctuation.

Before we relate the pairwise spin entanglement measure with the quantum fluctuation, we outline a behavior of the quantum fluctuation in a typical quantum spin chain. We employ the quantum Ising spin chain in a transverse magnetic field, which is a well-understood solvable spin model [3-5]. The Hamiltonian is given by $H=\sum_{j=1}^{N}\left[\sigma_{j}^{x} \sigma_{j+1}^{x}-h \sigma_{j}^{z}\right]$ with a transverse magnetic field $h(\geqslant 0)$ along the $z$ direction, where $\sigma^{x}$ and $\sigma^{z}$ are Pauli matrices. The spin number $N$ is infinite and we assume the cyclic boundary condition.

The property of the ground state $|\Psi\rangle$ depends substantially on $h$. In the limit of $h \rightarrow \infty$, all spin states are $|z+\rangle$, and $|\Psi\rangle$ is a product state $|\Psi\rangle_{h \rightarrow \infty}=\prod_{i}|z+\rangle_{i}$, which exhibits a paramagnetic (PM) phase forced by the transverse field. In the $x$ basis, $|\Psi\rangle_{h \rightarrow \infty}=\prod_{i}\left(|x+\rangle_{i}+|x-\rangle_{i}\right) / \sqrt{2}$ represents a random state, where there is no correlation between the fluctuations of the spins subject to the quantum uncertainty. If we gradually reduce the field strength $h$, the interaction term

*Corresponding author: shimizu.kaoru@lab.ntt.co.jp $\sigma_{j}^{x} \sigma_{j+1}^{x}$ results in a correlation between the spin fluctuations of neighboring spins. This paper identifies the quantum fluctuation by examining the correlation in the way specified above.

When we reduce $h$ close to unity, the correlation evolves in a singular way and the spin correlation length $\xi$ becomes long. Although the correlation is maximal, the staggered magnetization $M \equiv \sum_{i}(-1)^{i+1} \sigma_{j}^{x} / N$ remains zero on average and the system maintains the PM phase. At $h=1$, a spontaneous symmetry breakdown occurs and the system changes to the antiferromagnetic (AFM) phase, which is characterized by a finite staggered magnetic order $|\langle M\rangle|$ and a divergence in the spin correlation length $\xi$. This is a typical QPT occurring at absolute zero temperature. For $h \rightarrow$ 0 , the correlation gradually disappears and $|\Psi\rangle$ approaches $|\Psi \pm\rangle_{h=0}=|x \pm\rangle_{1}|x \mp\rangle_{2}|x \pm\rangle_{3}|x \mp\rangle_{4} \cdots$. At the limit of $h \rightarrow$ 0 , there is no correlation between the spin fluctuations of two spins in the $z$ basis and $\xi$ returns zero.

The above observation indicates that the QPT is governed by the emergence, evolution, and diminution of the correlation between the spin fluctuations of neighboring spins, where the correlation shows a singular maximum at the QPT point $h=1$ and decreases asymptotically to zero for both $h \rightarrow \infty$ and $h=0$. This is consistent with the general description of the QPT that is caused by a singular increase in the quantum fluctuation at the QPT point [5]. Since the spin fluctuation is driven by the quantum uncertainty, we must quantify the correlation using an appropriate quantum correlation measure for two spins. At first glance, the use of the concurrence $C(\rho)$, a measure of pairwise spin entanglement [6], seems straightforward as described in [2,3]. Here $\rho$ is the reduced density matrix calculated for a pair of neighboring spins $a$ and $b$ from the ground state $|\Psi\rangle$ of the spin chain in accordance with $\rho=\operatorname{Tr}_{\overline{a b}}[|\Psi\rangle\langle\Psi|]$, where the states of all other spins are averaged. Then we can compute $C(\rho)$ for $\rho$. Perfect and zero quantum correlation result in $C(\rho)=1$ and 0 , respectively.

However, the concurrence $C(\rho)$ does not exhibit the singular maximum at the QPT point $h=1[2,3]$ and cannot reproduce the quantum fluctuation behaviors observed in the QPT. We can explain the difference between $C(\rho)$ and the quantum 
fluctuation as follows. As the density matrix $\rho$ results from averaging over the quantum correlation $|\Psi\rangle=\sum_{i} c_{i}\left|f_{i}\right\rangle_{a b}\left|g_{i}\right\rangle_{\overline{a b}}$ between the spin pair $a b$ and the remainder $\overline{a b}$ of the spin chain, $C(\rho)$ decreases more when the spin correlation length $\xi$ is longer. This is the reason that $C(\rho)$ rather exhibits a small value at the QPT point $h=1$.

To remove the effect of the spatial coherence in $|\Psi\rangle$ on the pairwise spin entanglement, this paper focuses on the quantum correlation part $\rho_{q}=W \overline{\rho_{q}}$ embedded in $\rho$. We can determine $\rho_{q}$ by using the optimal Lewenstein-Sanpera (LS) decomposition of $\rho$ [7], where $W$ is the weight of $\rho_{q}$ and $\overline{\rho_{q}}$ is expressed by a pure entangled state of two spins as $\overline{\rho_{q}}=|\Phi\rangle\langle\Phi|$. Then, if the resulting $W$ and $|\Phi\rangle$ satisfy the nontrivial relationship $C(\rho)=W C(|\Phi\rangle)$, we can describe the quantum fluctuation properties with the pure entangled state $|\Phi\rangle$. Actually, $C(|\Phi\rangle)$ and $W$ exhibit the singular maximum and minimum at the QPT point $h=1$, respectively. Although we suggested the above statement numerically in the previous report [8], we have presented no proof so far.

In this paper, an analytic execution of the optimal LS decomposition of $\rho$ substantiates that (i) the spatial coherence effect on the pairwise spin entanglement can be incorporated into the $W$ part of the concurrence $C(\rho)$, and (ii) $C(|\Phi\rangle$ ) represents the quantum fluctuation amplitude relevant to the QPT and $|\Phi\rangle$ identifies the relative spin orientation characterizing the quantum fluctuation.

\section{ANALYTIC FORMULATION OF THE QUANTUM CORRELATION PART}

A transverse-field quantum Ising spin chain is a solvable spin chain model [3-5] that provides us the analytic expression for the quantum correlation part $\rho_{q}=W|\Phi\rangle\langle\Phi|$. This section formulates $W$ and $|\Phi\rangle$ in detail for further discussion in Sec. III.

\section{A. Finding quantum correlation part $\rho_{q}$ embedded in $\rho$}

First, we explain the general schemes. Once we obtain $\rho$ for a pair of $\frac{1}{2}$ spins, we can compute the concurrence using

$$
C(\rho)=\max \left\{0, c_{1}-\left(c_{2}+c_{3}+c_{4}\right)\right\},
$$

where $c_{i}$ represents the square root of the (real and positive) eigenvalues of the matrix $\rho\left[\left(\sigma^{y} \otimes \sigma^{y}\right) \rho^{*}\left(\sigma^{y} \otimes \sigma^{y}\right)\right]$ in decreasing order [6]. In accordance with Lewenstein and Sanpera [7], the density matrix $\rho$ can be decomposed into quantum $\rho_{q}=W \overline{\rho_{q}}$ and classical $\rho_{c}=(1-W) \overline{\rho_{c}}$ parts in a unique way as follows:

$$
\rho=(1-W) \overline{\rho_{c}}+W \overline{\rho_{q}} \quad \text { with } \quad \overline{\rho_{q}}=|\Phi\rangle\langle\Phi|,
$$

where (i) $\overline{\rho_{c}}$ and $\overline{\rho_{q}}$ are normalized, and (ii) the weight $W$ must be determined in such a way that $W$ exhibits the smallest possible value. This implies that the largest possible amount of quantum correlation is taken into account between the spin pair and the remainder of the system. In the following, we will refer to Eq. (2) as the optimal Lewenstein-Sanpera (LS) decomposition of $\rho$.

The classical part $\rho_{c}$ contains only the classical correlation given by $\sum_{i} p_{i}\left(\rho_{a} \otimes \rho_{b}\right)_{i}$ with the probabilities $p_{i}$ of some different separable two-spin states $\left(\rho_{a} \otimes \rho_{b}\right)_{i}$. By contrast, the quantum part $\overline{\rho_{q}}$ is a pure entangled state $|\Phi\rangle=\{s, t, u, v\}$ of two spins with a $\{|z \pm\rangle\}$ basis:

$$
\begin{aligned}
|\Phi\rangle= & s|z+, z+\rangle_{a b}+t|z+, z-\rangle_{a b} \\
& +u|z-, z+\rangle_{a b}+v|z-, z-\rangle_{a b} \\
& \text { with }|s|^{2}+|t|^{2}+|u|^{2}+|v|^{2}=1 .
\end{aligned}
$$

We can also compute the concurrence $C(|\Phi\rangle)$ for $\overline{\rho_{q}}(=|\Phi\rangle\langle\Phi|)$ as

$$
C(|\Phi\rangle)=2|s v-t u| \leqslant 1 .
$$

Here we should note that $C(|\Phi\rangle)$ is not invariant for local spin rotations on $\rho$ and not an entanglement measure defined for $\rho$. Property of the quantity $W C(|\Phi\rangle)$ is examined [9] and the equality $C(\rho)=W C(|\Phi\rangle)$ does not always hold in general [10]. However, there are some nontrivial cases that lead to the equality as we discuss in this paper.

To execute the decomposition, we use the trial density matrix $\rho_{\text {trial }}$ below:

$$
\rho_{\text {trial }}=\rho-W|\Phi\rangle\langle\Phi| .
$$

Wellens and Kuś established an algebraic method for finding the optimal LS decomposition of $\rho$ when $\operatorname{rank}(\rho)=4$. They derived the conditions and criterion of the optimal LS decomposition as theorem 1 in the article [10].

The first condition is relevant to the fact that $\rho_{\text {trial }}$ must be separable. Let us introduce the parameter space spanned by $\{s, t, u, v\}$. The space is divided into two different regions, I and II, where trial density matrices $\rho_{\text {trial }}$ are separable and inseparable, respectively. Figure 1 shows schematics that illustrate the searching for a certain set $\{s, t, u, v\}$ that minimizes $W$ while retaining the separable nature for $\rho_{\text {trial }}$. First, we introduce the initial set $\left\{s_{0}, t_{0}, u_{0}, v_{0}\right\}$ such that $\rho_{\text {trial }}$ is a separable density matrix and in region I. Then we change the set slightly so that the weight $W$ can be smaller. However, if we change $\{s, t, u, v\}$ too much, $\rho_{\text {trial }}$ enters the inseparable region,

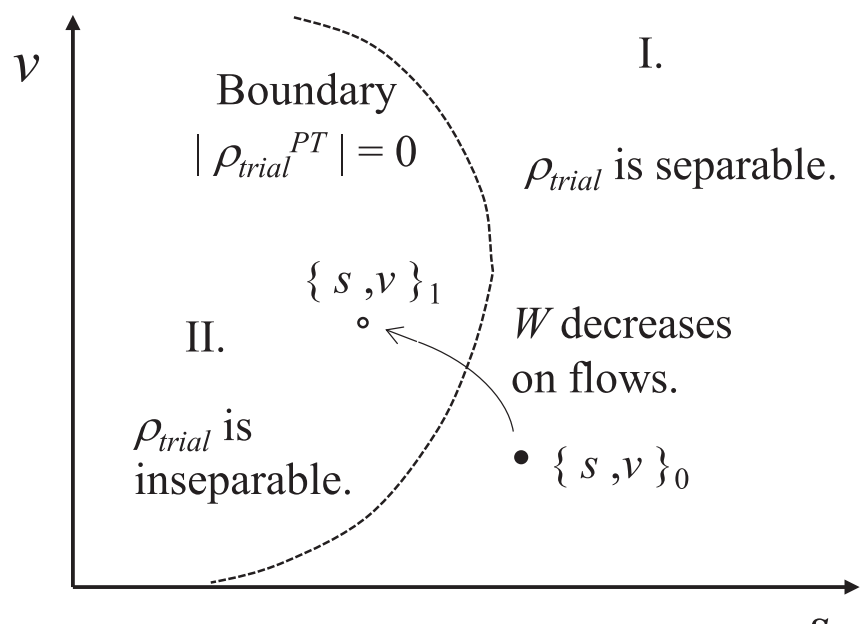

$S$

FIG. 1. Schematics for determining the optimal LS decomposition of $\rho$ with the minimal weight $W$ are illustrated. The parameter space is simplified to a two-dimensional space spanned with $\{s, v\}$. In regions I and II, the trial density matrices $\rho_{\text {trial }}$ are separable and inseparable, respectively. If too great a change is made in $\{s, v\}, \rho_{\text {trial }}$ enters region II, which is undesired. Therefore, the candidates of $\{s, v\}$ are located at the boundary between regions I and II. Then we seek $\{s, v\}$ that minimizes $W$ at the boundary. 
which defeats our purpose. Therefore, we can find $\{s, t, u, v\}$ candidates at the boundary between regions I and II, where the boundary itself belongs to region I. At the boundary, we search for $\{s, t, u, v\}$ that minimizes $W[10]$.

We can examine whether or not $\rho_{\text {trial }}$ is separable as $\sum_{i} p_{i}\left(\rho_{a} \otimes \rho_{b}\right)_{i}$ using the Peres criterion [11]. The criterion is based on an eigenvalue evaluation of the modified density matrix $\sigma^{\mathrm{PT}}$ (partial transpose of $\sigma$ ) given by

$$
\sigma^{\mathrm{PT}}=\left[\begin{array}{llll}
\sigma_{11} & \sigma_{12} & \sigma_{31} & \sigma_{32} \\
\sigma_{21} & \sigma_{22} & \sigma_{41} & \sigma_{42} \\
\sigma_{13} & \sigma_{14} & \sigma_{33} & \sigma_{34} \\
\sigma_{23} & \sigma_{24} & \sigma_{43} & \sigma_{44}
\end{array}\right]
$$

The partial transpose (PT) reverses the time evolution of either spin. If and only if the two spins are separable, $\sigma^{\mathrm{PT}}$ can represent another physical system and all the eigenvalues of $\sigma^{\mathrm{PT}}$ are non-negative. By contrast, when the two spins are entangled, $\sigma^{\mathrm{PT}}$ corresponds to no physical system and must have at least one negative eigenvalue [10]. Therefore, we can specify the boundary by stipulating two requirements for $\rho_{\text {trial }}^{\mathrm{PT}}$, namely that (i) all the eigenvalues of $\rho_{\text {trial }}^{\mathrm{PT}}$ must be non-negative and (ii) at least one of them must be zero. Hence, finally, we can formulate the condition as

$$
\operatorname{rank}\left(\rho_{\text {trial }}^{\mathrm{PT}}\right)=3 \rightarrow\left|\rho_{\text {trial }}^{\mathrm{PT}}\right|=0 \text {, i.e., } \exists_{|\phi\rangle} \rho_{\text {trial }}^{\mathrm{PT}}|\phi\rangle=0 .
$$

When the pure entangled state $|\Phi\rangle$ of two spins [see Eq. (2)] is not always a maximally entangled state, the second condition of the optimal LS decomposition is given by the set of two requirements below [10]:

(a) $\operatorname{rank}\left(\rho_{\text {trial }}\right)=3 \rightarrow\left|\rho_{\text {trial }}\right|=0$, i.e., $\exists_{|\tilde{\phi}\rangle} \rho_{\text {trial }}|\tilde{\phi}\rangle=0$,

and

(b) $\hat{M}|\Phi\rangle=\lambda|\Phi\rangle$ with $\lambda \leqslant 0$,

where $\hat{M}$ is introduced as follows using $|\phi\rangle$ [see Eq. (6)] and $|\tilde{\phi}\rangle$ [see Eq. (7)]:

$$
\hat{M}=\mu|\tilde{\phi}\rangle\langle\tilde{\phi}\rangle\left|+[|\phi\rangle\langle\phi\rangle \mid]^{\mathrm{PT}} \text { with } \mu \geqslant 0 .\right.
$$

The proof is presented in Ref. [10]. When $\rho$ is a blockdiagonal density matrix with the $\operatorname{rank}(\rho)=4$, we can find and confirm the optimal decomposition in an analytic way using Eqs. (6)-(9) as explained in Appendix B.

\section{B. Example: A transverse-field quantum Ising spin chain in the PM phase}

We examine the PM phase $(h>1)$ of the ground state for a transverse-field quantum Ising spin chain [3], where the analytic solution of $|\Psi\rangle$ is available. The density matrix $\rho$ of the neighboring spins is represented by a block-diagonal matrix with the basis $\left\{|z+, z+\rangle_{a b},|z+, z-\rangle_{a b},|z-, z+\rangle_{a b},|z-, z-\rangle_{a b}\right\}$,

$$
\rho(h)=\left[\begin{array}{cccc}
A & 0 & 0 & B \\
0 & E & F & 0 \\
0 & F & E & 0 \\
B & 0 & 0 & D
\end{array}\right] .
$$

All the elements are real and expressed as different functions of $h(\geqslant 1)$ as shown in Appendix A [3]. All the diagonal elements are positive and their sum is unity. $B$ and $F$ are negative. The other elements exhibiting zero value indicate the absence of the staggered magnetization $M \equiv$ $\sum_{i}(-1)^{i+1} \sigma_{j}^{x} / N$. As the rank of $\rho(h)$ is 4 , we can utilize the method established by Wellens and Kuś [10]. For all the finite $h(\geqslant 1)$ value, $A D-|B|^{2}>0, E^{2}-|F|^{2}>0$, and $\sqrt{A D}+|B|>E+|F|$ hold. For example, when $h=1$, the values $A, E, D, B$, and $F$ are $0.703405,0.114905,0.066785$, -0.212207 , and -0.106103 , respectively. In the PM phase, the concurrence $C(\rho)$ [see Eq. (1)] is always given by

$$
C(\rho)=2(|B|-E) .
$$

In the following, we find the optimal decomposition of $\rho$ [see Eq. (2)] using Eqs. (6) and (7). As $\{s, t, u, v\}$ are real, the trial density matrix $\rho_{\text {trial }}(=\rho-W|\Phi\rangle\langle\Phi|)$ is

$$
\rho_{\text {trial }}=\left[\begin{array}{cccc}
A-W s^{2} & -W s t & -W s u & B-W s v \\
-W s t & E-W t^{2} & F-W t u & -W t v \\
-W s u & F-W t u & E-W u^{2} & -W u v \\
B-W s v & -W t v & -W u v & D-W v^{2}
\end{array}\right] .
$$

The condition $\left|\rho_{\text {trial }}^{\mathrm{PT}}\right|=0$ [see Eq. (6)] results in the relationship $f\left(s^{2}, t^{2}, u^{2}, v^{2}, s v, t u\right)=0$. Here $f$ is a function of $s^{2}, t^{2}, u^{2}, v^{2}, s v$, and $t u$. This relationship indicates that both $\{s, t, u, v\}$ and $\{s,-t,-u, v\}$ are candidates for $|\Phi\rangle$. However, this contradicts the uniqueness of the decomposition. Hence, $(t, u)=(0,0)$ when $(s, v) \neq(0,0)$. In a similar way, $(s, v)=(0,0)$ when $(t, u) \neq(0,0)$. As $|\Phi\rangle=|z+, z+\rangle_{a b}$ holds for $h \rightarrow \infty$, we choose $(s, v) \neq(0,0)$ and $(t, u)=$ $(0,0)$. Hence, the two-spin state $|\Phi\rangle$ is $|\Phi\rangle=\{s, 0,0, v\}=$ $s|z+, z+\rangle_{a b}+v|z-, z-\rangle_{a b}$ and $C(|\Phi\rangle)=2|s v|$ holds [see Eq. (4)], where we take $s$ positive.

Since $(t, u)=(0,0)$ holds, $\rho_{\text {trial }}$ and $\rho_{\text {trial }}^{\mathrm{PT}}$ can be simplified as

$$
\rho_{\text {trial }}=\left[\begin{array}{cccc}
A-W s^{2} & 0 & 0 & B-W s v \\
0 & E & F & 0 \\
0 & F & E & 0 \\
B-W s v & 0 & 0 & D-W v^{2}
\end{array}\right],
$$

and

$$
\rho_{\text {trial }}^{\mathrm{PT}}=\left[\begin{array}{cccc}
A-W s^{2} & 0 & 0 & F \\
0 & E & B-W s v & 0 \\
0 & B-W s v & E & 0 \\
F & 0 & 0 & D-W v^{2}
\end{array}\right],
$$

respectively. The condition $\left|\rho_{\text {trial }}^{\mathrm{PT}}\right|=0$ [see Eq. (6)] results in the relationship

$$
\text { (i) }(B-W s v)^{2}-E^{2}=0 \text {, }
$$

or

$$
\text { (ii) }\left(A-W s^{2}\right)\left(D-W v^{2}\right)-F^{2}=0 \text {. }
$$

When we choose case (i), we can obtain the optimal LS decomposition. Since $B$ is negative $(B<0)$, we obtain the 
relationship below from Eq. (15):

$$
W 2|s v|=2(|B|-E)>0,
$$

where $s>0$ and $v<0$. The condition $\left|\rho_{\text {trial }}\right|=0$ [see Eq. (7)] and Eq. (15) leads to

$$
\left(A-W s^{2}\right)\left(D-W v^{2}\right)-E^{2}=0 .
$$

Moreover, we can utilize the normalization condition:

$$
s^{2}+v^{2}=1 \text {. }
$$

Appendix B confirms that the set of equations (17)-(19) satisfies the requirements, Eqs. (8) and (9), for the optimal LS decomposition when $|\Phi\rangle=\{s, 0,0, v\}$ with $s>0$ and $v<0$.

Therefore, we can find the decomposition using Eqs. (17)(19). We can determine three independent variables $W$ and $\{s, v\}$ by solving Eqs. (17)-(19). Finally, as outlined in Appendix $\mathrm{C}$, we can obtain the analytic solution for $W$ as follows by introducing $X=|B|-E>0$ :

$$
\begin{aligned}
W= & \frac{A+D}{2}\left[1-\frac{(E+X)(E-X)}{A D}\right] \\
& -\frac{A-D}{2} \sqrt{\left(1-\frac{(E+X)^{2}}{A D}\right)\left(1-\frac{(E-X)^{2}}{A D}\right)} .
\end{aligned}
$$

The two-spin state $|\Phi\rangle=\{s, 0,0, v\}$ is expressed with

$$
\begin{aligned}
& s=\frac{1}{2}\left(\sqrt{1-\frac{2 X}{W}}+\sqrt{1+\frac{2 X}{W}}\right)>0 \text { and } \\
& v=\frac{1}{2}\left(\sqrt{1-\frac{2 X}{W}}-\sqrt{1+\frac{2 X}{W}}\right)<0 .
\end{aligned}
$$

Thus, the block-diagonal form of the legitimate density matrix $\rho$ [see Eq. (10)] makes it possible to execute the optimal LS decomposition in an analytic way. Moreover, by comparing Eq. (11) with Eq. (17) and using Eq. (4), we can show the equality

$$
C(\rho)=W 2|s v|=W C(|\Phi\rangle)
$$

for the employed spin model. In accordance with the article [10], the equality $C(\rho)=W C(|\Phi\rangle)$ does not always hold when the $\operatorname{rank}\left(\rho_{\text {trial }}\right)=3$. Hence, there is a nontrivial physical reason that leads to the equality. The equality also holds for the antiferromagnetic (AFM) phase that is $0 \leqslant h \leqslant 1$. This is self-evident from the fact that $C(\rho), W$, and $C(|\Phi\rangle)$ are continuous functions of $h$ at the QPT point $h=1$.

\section{DISCUSSION}

By using the analytic solutions, this section substantiates that (i) $W$ is relevant to the spatial coherence effect on the pairwise spin entanglement and (ii) $|\Phi\rangle$ is relevant to the relative spin orientation characterizing the quantum fluctuation. Then we discuss the validity of our framework for more general spin models.

\section{A. Physical counterparts of the weight $W$ and the two-spin state $|\Phi\rangle$}

An arbitrary spin pair cannot monopolize a large concurrence value $C(\rho)(<1)$ when $n$ spins are entangled and the

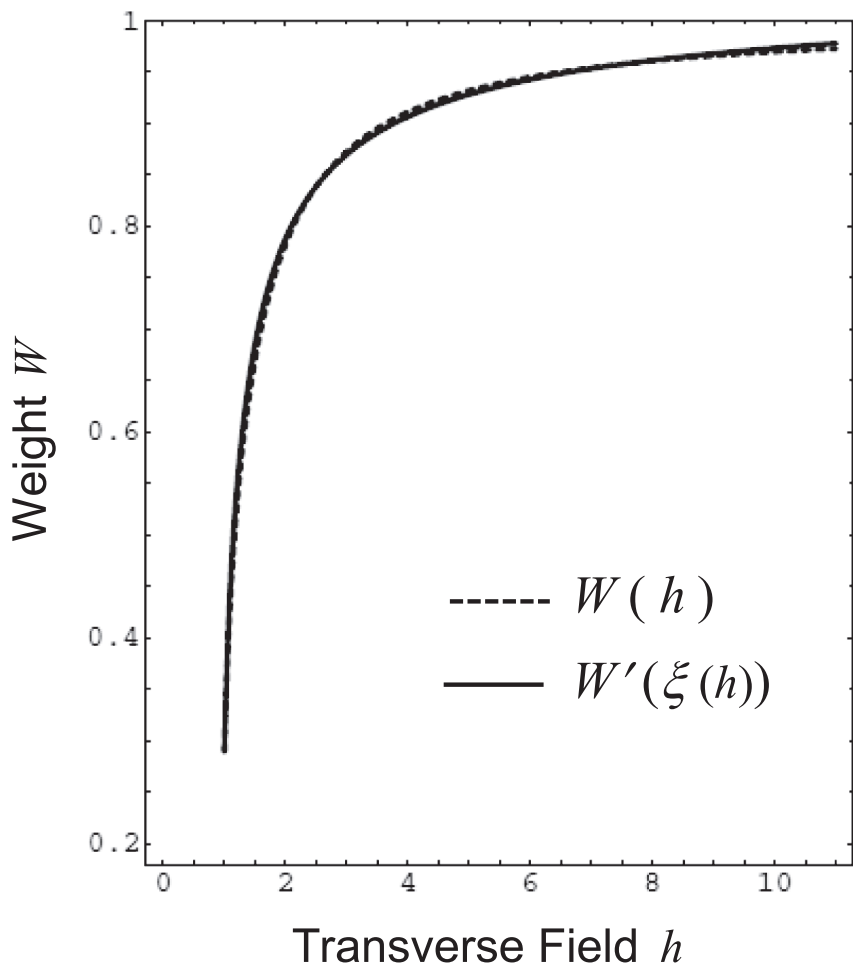

FIG. 2. The dashed and plain curves, respectively, show the analytic solution of the weight $W(h)$ obtained using Eq. (20) and the result for $W^{\prime}[\xi(h)]$ obtained using Eq. (25) with respect to the field strength from $h=1$ to $h=11$. To compute $W^{\prime}[\xi(h)], \xi(h)=$ $1 /\left(2 \ln _{\mathrm{e}} h\right)$ is substituted into $\xi$ as a function of the field strength $h$.

concurrence $C(\rho)$ is shared or diluted by $n$ spins. This is a general effect called a shared entanglement [1]. This section shows that we can attribute the weight $W(h)$ to the shared entanglement effect on $C(\rho)$. First, we outline two typical examples of shared entanglement. (i) If state $|\Psi\rangle$ of $n$ spins is represented by an equally weighted superposition of all the possible $n !(p ! q !)^{-1}$ product states of $(|z+\rangle)^{\otimes p}$ and $(|z-\rangle)^{\otimes q}$ with $n=p+q$, the concurrence $C(\rho)$ of an arbitrary spin pair is related to $n$ by $C(\rho) \sim a / n$ [12]. (ii) If $n$ spins form a closed spin chain and $|\Psi\rangle$ is the ground state of the spin chain for a fictitious Hamiltonian that assumes a conservation of $\sum_{i} \sigma_{i}^{z}$ and a nearest-neighbor spin-spin interaction excluding $|z+, z+\rangle$, the concurrence $C(\rho)$ of two neighboring spins is related to $n$ by $C(\rho) \sim a+b / n^{2}$ for a large $n$ limit [13]. In both cases, an increase in the number $n$ of entangled spins reduces the $C(\rho)$ value. This is exactly the effect of the spatial quantum coherence in the spin chain on the pairwise spin entanglement. Thus, shared entanglement is a common characteristic of the entangled states of many spins. Different spin models provide different relationships between $C(\rho)$ and $n$.

Our aim is to confirm the shared entanglement in a transverse-field quantum Ising spin chain. As elements $A-F$ of $\rho$ [see Eq. (10)] are given as a function of the transverse-field strength $h$, we can compute $W(h)$ with respect to $h$ in the PM phase using Eq. (20). The dashed curve in Fig. 2 shows the analytic solution of $W(h)$, where the $h$ value is from 1 to 11 . The weight $W$ exhibits a singular minimum of 0.289913 at the QPT point $(h=1)$ and increases asymptotically to unity in the limit of $h \rightarrow \infty$. In the employed spin model, a change 
in the field strength $h$ from 1 to infinity $(\infty)$ shortens the spin correlation length $\xi$ from infinity $(\infty)$ to a small cutoff $[4,5]$. Since $\xi$ means the size of a cluster composed of entangled spins, the observed $W(h)$ behavior is consistent to the shared entanglement effect, if we assume the unity lattice constant.

To find the shared entanglement relationship, we analyze the asymptotic behavior of $W(h)$ at just above the QPT point $h=1$, where we introduce $\eta$ as a small positive deviation of $h=1+\eta$ from 1 . From the analytic solution of $W(h)$ [see Eq. (20)] just above $h=1$, we can derive the asymptotic behavior of $W(h)$ valid only for an infinitely small deviation $\eta$,

$$
W(h)=W(1+\eta)=\alpha+\beta \eta \ln _{\mathfrak{e}}(1 / \eta),
$$

with

$$
(\alpha, \beta)=(0.289913,0.444433) .
$$

Here the logarithmic divergence of the elliptic integral is taken into account as shown in Appendix A. On the other hand, the spin correlation length $\xi$ is represented by $\xi(h)=1 /\left(2 \ln _{\mathrm{e}} h\right)$ with $h>1$, where we can find an $e^{-R / \xi}$ term in the spin correlation function: $\left\langle s_{i}^{Z} s_{i+R}^{Z}\right\rangle-1 / 4 \approx$ $-(1 / h)^{2 R} O\left(R^{-2}\right)$ of the $\sigma^{z}$ component [14]. $\xi=1 /(2 \eta)$ holds for $\eta \ll 1$ and $\xi$ is divergent at $\eta \rightarrow 0$. Therefore, we can relate the weight $\widetilde{W^{\prime}}(\xi)$ directly to the correlation length $\xi$ as

$$
\widetilde{W}^{\prime}(\xi)=\alpha+\beta\left(\frac{1}{2 \xi}\right) \ln _{\mathrm{e}}(2 \xi)
$$

The $\ln _{\mathrm{e}}(2 \xi)$ term on the right-hand side reminds us of block entanglement entropy $[1,15]$.

Equation (24) is valid for a sufficiently long $\xi$. However, in the limit of $h \rightarrow \infty, \xi$ decreases asymptotically close to zero and the $(2 \xi)^{-1}$ term in Eq. (24) is divergent. To avoid this divergence and test the shared entanglement relationship that governs all the PM phase $(1<h<\infty)$, we modify Eq. (24) as follows by replacing $\xi$ with $\frac{1}{2}+\xi$ :

$$
\begin{aligned}
W^{\prime}(\xi) & =\alpha+\beta\left[\frac{1}{2\left(\frac{1}{2}+\xi\right)}\right]\left\{\ln _{\mathrm{e}}\left[2\left(\frac{1}{2}+\xi\right)\right]+\gamma\right\} \text { with } \\
\gamma & =1.845
\end{aligned}
$$

where $\gamma$ is a fitting parameter. Equation (25) gives us Eq. (24) in the limit of $\xi \rightarrow \infty$. The weight $W^{\prime}(\xi)$ depends on the field strength $h$ in an indirect way mediated by the $h$ dependence of the correlation length $\xi(h)=1 /\left(2 \ln _{\mathrm{e}} h\right)$. Hence, we can compare the two different weight expressions $W(h)$ [see Eq. (20)] and $W^{\prime}[\xi(h)]$ [see Eq. (25)]. If and only if the weight $W$ implies shared entanglement, $W^{\prime}[\xi(h)]$ agrees with $W(h)$ over a wide region of $h \geqslant 1$. Otherwise $W$ is irrelevant to the shared entanglement.

The plain curve in Fig. 2 shows the $W^{\prime}[\xi(h)]$ result obtained using Eq. (25). As shown in Fig. 2, $W^{\prime}(\xi)$ reproduces exactly the analytic solution $W(h)$ over $h=1$ to 11 , where the definition $\xi(h)=1 /\left(2 \ln _{\mathrm{e}} h\right)$ loses validity if $h$ is far larger than 10 and $\xi(h) \ll 1$. The observed agreement between $W(h)$ and $W^{\prime}[\xi(h)]$ evidences that $W$ indicates the shared entanglement of the concurrence $C(\rho)$. Therefore, the effect of the spin correlation length $\xi$ on $C(\rho)$ is completely separated into the $W$ part of the factorization $C(\rho)=W(\xi) C(|\Phi\rangle)$ [see Eq. (22)]. Moreover, $W^{\prime}(\xi)$ [see Eq. (25)] represents the

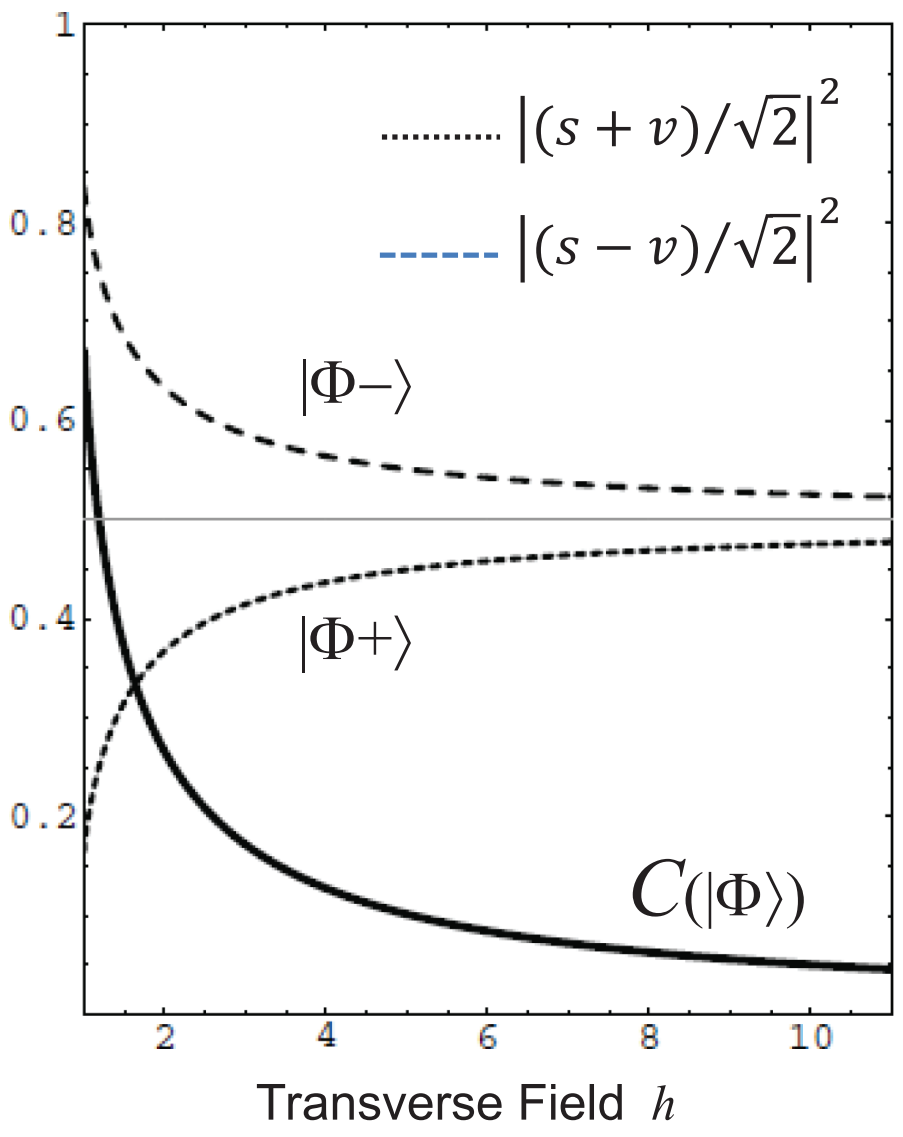

FIG. 3. (Color online) The bold curve shows the evolution of the quantum fluctuation amplitude $C(|\Phi\rangle)$ with respect to the reduction in the field strength from $h=11$ to $h=1$. The dashed and dotted curves represent the squared amplitudes of the $|\Phi-\rangle$ and $|\Phi+\rangle$ components. $|\Phi-\rangle$ is advantageous as regards energy cost.

shared entanglement relationship between $W$ and $\xi$, which characterizes the PM phase of the transverse-field quantum Ising spin chain.

Since the entire effect of the spin correlation length $\xi$ on $C(\rho)$ is separated into the $W$ part, we can consider the $C(|\Phi\rangle)$ part to be relevant to the relative spin orientation of two neighboring spins, that is, the correlation between the spin fluctuations, which we define as the quantum fluctuation. We can examine the structure of the quantum fluctuation using $|\Phi\rangle=s|z+, z+\rangle_{a b}+v|z-, z-\rangle_{a b}$ [see Eq. (21)] and evaluate its amplitude $C(|\Phi\rangle)=2|s v|$ [see Eq. (4)]. For example, at $h \rightarrow \infty,|\Phi\rangle \approx|z+, z+\rangle_{a b}$ holds with $s=1$, and $C(|\Phi\rangle)$ approaches asymptotically zero.

Figure 3 shows the change in the two-spin state $|\Phi\rangle\left(=s|z+, z+\rangle_{a b}+v|z-, z-\rangle_{a b}\right)$ with respect to the reduction in the transverse field $h$ from 11 to 1 in the PM phase. By using $|\Phi \pm\rangle=(|z+, z+\rangle \pm|z-, z-\rangle) / \sqrt{2}$, we can rewrite $|\Phi\rangle=\{(s+v) / \sqrt{2}\}|\Phi+\rangle+\{(s-v) / \sqrt{2}\}|\Phi-\rangle$. When $h$ decreases from infinity $(\infty)$, an imbalance between $|\Phi+\rangle$ and $|\Phi-\rangle$ leads to a finite correlation. This is because the interaction term $\sigma_{j}^{x} \sigma_{j+1}^{x}$ decreases and increases the energy costs of the antiferromagnetic fluctuation $|\Phi-\rangle=$ $(|x+, x-\rangle+|x-, x+\rangle) / \sqrt{2}$ and the ferromagnetic fluctuation $|\Phi+\rangle=(|x+, x+\rangle+|x-, x-\rangle) / \sqrt{2}$, respectively. The former 
is advantageous as regards energy cost of the spin pair. Hence, the relative amplitudes of $|\Phi-\rangle$ and $|\Phi+\rangle$ are enhanced and reduced, respectively. At $h=1$ (the QPT point), $C(|\Phi\rangle)$ exhibits a singular maximum. Thus we can describe the dynamics of the spin chain in terms of the quantum fluctuation using the two-spin state $|\Phi\rangle$. The energy cost estimation for the spin pair using $|\Phi\rangle$ makes sense if and only if a two-body spin interaction governs the dynamics of the spin system.

\section{B. Is the framework valid for a general spin model?}

Although we derived the equality $C(\rho)=W(\xi) C(|\Phi\rangle)$ [see Eq. (22)] from a specific solvable spin chain model, namely, a transverse-field quantum Ising spin chain, the results obtained for $W(\xi)$ and $|\Phi\rangle$ expressed typical properties common to a general spin chain model. In particular, the shared entanglement of the concurrence $C(\rho)$ must be a common characteristic of a general spin chain model if the model has a translational symmetry and accordingly a well-defined spin correlation length $\xi$. Moreover, when the system is governed by a nearest-neighbor two-body spin interaction as with the Ising-type interaction, the two-spin state $|\Phi\rangle$ is sufficient to determine the energy cost of the spin pair. Hence, our framework for determining $W$ and $|\Phi\rangle$ seems valid for a general spin chain model which is characterized by translational symmetry and a nearest-neighbor two-body spin interaction.

From a mathematical viewpoint, the equality $C(\rho)=$ $W C(|\Phi\rangle)$ holds if and only if the rank $R$ of the quantum correlation part $\rho_{q}$ is really $R=1$. An arbitrary two-spin density matrix $\rho$ does not always satisfy this condition and leads to the inequality $C(\rho) \leqslant W C(|\Phi\rangle)[10]$. However, if the rank $R$ of $\rho_{q}$ is guaranteed being $R=1$ because of a legitimate physical reason specific to $\rho$, it is self-evident that the optimal LS decomposition $\rho=(1-W) \overline{\rho_{c}}+W|\Phi\rangle\langle\Phi|$ yields the equality $C(\rho)=W C(|\Phi\rangle)$. Actually, $\rho$ of our present interest is specific to a density matrix $\rho=\operatorname{Tr} \frac{a b}{a b}[|\Psi\rangle\langle\Psi|]$, which is reduced from the ground state $|\Psi\rangle$ of a spin chain model that is characterized by a nearest-neighbor two-body spin interaction.

To observe the effect of a nearest-neighbor two-body spin interaction on the resulting density matrix $\rho$, we first assume a case where $N=2$. We can always determine the two-spin state $|\Phi\rangle=|\Phi\rangle^{(2)}$ that is identical to the ground state $|\Psi\rangle$ and the rank of $\rho_{q}=(|\Phi\rangle\langle\Phi|)^{(2)}$ is always $R=1$. Then we examine how the quantum correlation part $\rho_{q}$ changes with respect to the increase in the spin number $N$. In the Ising-type interaction case analyzed in Sec. III B, the increase in $N$ renews the coefficients $\{s, t, u, v\}$ of the two-spin state $|\Phi\rangle^{(N)}$ while retaining the $\operatorname{rank} R=1$ of $\rho_{q}$ as $\rho_{q}^{(N)} \propto(|\Phi\rangle\langle\Phi|)^{(N)}$.

The detailed difference in the two-body spin interaction form only influences the relative spin orientation optimizing the energy cost and does not alter the way in which $\rho_{q}$ changes from $\rho_{q}^{(2)} \propto(|\Phi\rangle\langle\Phi|)^{(2)}$ to $\rho_{q}^{(N)}$ with keeping $\operatorname{rank}\left(\rho_{q}\right)=1$. Hence, we can postulate that the rank of $\rho_{q}$ is guaranteed being $R=1$ and the equality $C(\rho)=W C(|\Phi\rangle)$ always holds when a spin chain model is characterized by a nearestneighbor two-body spin interaction and translational symmetry. We must undertake further studies to examine whether or not the framework is valid for a more general spin model as regards the interaction range, dimension, and spatial inhomogeneity of the system. Regardless of the dimension and the interaction range, the shared entanglement of $C(\rho)$ is a substantial property of all the spin models. However, it is not always possible to assume the $\operatorname{rank}\left(\rho_{q}\right)=1$ a priori.

Finally, we mention the practical aspects of the optimal LS decomposition of $\rho$. The decomposition generally requires a very complicated numerical search for determining $\{s, t, u, v\}$. Fortunately, this is not always true. Suppose that (i) $\rho(\chi)$ is a function of the external parameter $\chi$ such as a field strength $h$, and (ii) the analytic solution $\left\{s_{0}, t_{0}, u_{0}, v_{0}\right\}$ is available for $\rho\left(\chi_{0}\right)$ when $\chi=\chi_{0}$. In such a case, we can search appropriate $\{s, t, u, v\}$ near around $\left\{s_{0}, t_{0}, u_{0}, v_{0}\right\}$ in a numerical way for a renewed density matrix $\rho\left(\chi_{0}+\delta \chi\right)$ that is slightly different from $\rho\left(\chi_{0}\right)$ with a small deviation $\delta \chi$ from $\chi_{0}$. By repeating the above procedure in a recursive way, we can compute $\{s, t, u, v\}$ for all $\rho(\chi)$. The initial analytic solution is available if $\rho\left(\chi_{0}\right)$ is a block-diagonal matrix or represented by a product state. Actually, we executed the decomposition of $\rho$ at the antiferromagnetic (AFM) phase $(0 \leqslant h<1)$ of the transverse-field quantum Ising spin chain with the numerical method mentioned above, where the QPT point $h=1$ provided the initial solution $\left\{s_{0}, t_{0}, u_{0}, v_{0}\right\}$. The results are shown in the previous report [8].

\section{SUMMARY}

This paper quantified the quantum fluctuation properties of a quantum spin chain subject to a quantum phase transition (QPT) by introducing an appropriate pairwise entanglement measure for neighboring two spins. The concurrence $C(\rho)$ for the reduced density matrix $\rho$ of the two spins was inappropriate to this purpose. Actually, $C(\rho)$ did not exhibit a singular increase in the quantum fluctuation amplitude that was observed necessarily at the QPT point. This was because the spatial coherence spread over the spin chain rather decreased $C(\rho)$ at the QPT point.

Instead of $C(\rho)$, we were able to describe the quantum fluctuation properties with the normalized quantum correlation part $\overline{\rho_{q}}=|\Phi\rangle\langle\Phi|$ embedded in $\rho$. Here the optimal Lewenstein-Sanpera (LS) decomposition of $\rho$ determined the weight $W$ of the quantum correlation part $W \overline{\rho_{q}}$ and the pure entangled state $|\Phi\rangle$ of two spins. The concurrence $C(|\Phi\rangle)$ for $|\Phi\rangle$ represented the quantum fluctuation amplitude and $|\Phi\rangle$ identified the relative spin orientation characterizing the quantum fluctuation. The spatial coherence effect on the pairwise spin entanglement was incorporated into the $W$ part of the nontrivial relationship for the concurrence $C(\rho)=$ $W C(|\Phi\rangle)$. The analytic execution of the decomposition with a solvable spin model, a transverse-field quantum Ising spin chain, substantiated the above statements completely. These statements were valid to a more general spin chain model that was characterized by a nearest-neighbor two-body spin interaction and translational symmetry.

\section{ACKNOWLEDGMENT}

The authors thank Dr. A. Kawaguchi for his contribution to our early studies and Dr. F. Morikoshi for his fruitful comments.

\section{APPENDIX A}

For the PM phase $(h>1)$ of the transverse-field quantum Ising spin chain $[3,4]$, the elements of the reduced density 
matrix $\rho$ for two neighboring spins are given by

$$
\begin{aligned}
& A=1 / 4+G_{0} / 2+\left(G_{0}^{2}-G_{-1} G_{+1}\right) / 4, \\
& E=H=1 / 4-\left(G_{0}^{2}-G_{-1} G_{+1}\right) / 4, \\
& D=1 / 4-G_{0} / 2+\left(G_{0}^{2}-G_{-1} G_{+1}\right) / 4, \\
& B=\left(G_{-1}-G_{+1}\right) / 4, \quad \text { and } F=\left(G_{-1}+G_{+1}\right) / 4 .
\end{aligned}
$$

With the first $K(k)$ and second $E(k)$ elliptic integrals, $\left\{G_{0}, G_{-1}, G_{+1}\right\}$ are given by

$$
\begin{aligned}
G_{0}= & {[(1-l) / \pi] K(k)+[(1+l) / \pi] E(k), } \\
G_{-1}= & {[(1-l) / l \pi] K(k)+[(1+l) / l \pi] E(k), } \\
G_{+1}= & {\left[(1-l)\left(2 l^{2}+1\right) / 3 l \pi\right] K(k) } \\
& +\left[(1+l)\left(2 l^{2}-1\right) / 3 l \pi\right] E(k),
\end{aligned}
$$

with $k^{2}=4 l /(1+l)^{2} \leqslant 1$ and $l=1 / h$. The elliptic integrals are expressed as

$$
\begin{aligned}
& K(k) \equiv \int_{0}^{\pi / 2} d \varphi 1 / \sqrt{1-k^{2} \sin ^{2} \varphi}, \quad \text { and } \\
& E(k) \equiv \int_{0}^{\pi / 2} d \varphi \sqrt{1-k^{2} \sin ^{2} \varphi} .
\end{aligned}
$$

$K(k)$ exhibits the logarithmic divergence below:

$$
\begin{aligned}
K(k) & =K\left(\sqrt{1-\eta^{2} / 4}\right) \\
& \sim 1.3865+0.5 \ln _{\mathrm{e}}(1 / \eta) \text { for } \eta \rightarrow+0,
\end{aligned}
$$

whereas $E(k)$ becomes $1+O\left(\eta^{2}\right)$ for $\eta \rightarrow+0$.

\section{APPENDIX B}

In accordance with theorem 1 in the article by Wellens and Kuś [10], we can decide whether or not a given decomposition $(1-W) \overline{\rho_{c}}+W|\Phi\rangle\langle\Phi|$ of $\rho$ is optimal with the minimum $W$ using Eqs. (6)-(9). First, we find $|\tilde{\phi}\rangle$ and $|\phi\rangle$ satisfying $\rho_{\text {trial }}|\tilde{\phi}\rangle=0$ [see Eq. (7)] and $\rho_{\text {trial }}^{\mathrm{PT}}|\phi\rangle=0$ [see Eq. (6)], respectively. We can solve $s^{2}$ and $v^{2}$ as the functions of $\{A, D, E, W\}$ using Eqs. (18) and (19). If we replace $B-W s v$ with $-E$, we can rewrite $\rho_{\text {trial }}$ [see Eq. (13)] as follows after eliminating $s^{2}$ and $v^{2}$ :

$$
\rho_{\text {trial }}=\left[\begin{array}{cccc}
(1+\sqrt{1-\Gamma}) E / \sqrt{\Gamma} & 0 & 0 & -E \\
0 & E & F & 0 \\
0 & F & E & 0 \\
-E & 0 & 0 & (1-\sqrt{1-\Gamma}) E / \sqrt{\Gamma}
\end{array}\right],
$$

where $\Gamma=4 E^{2} /(A+D-W)^{2}$ and $0<\Gamma<1$. Hence, we can choose $|\tilde{\phi}\rangle$ and $|\phi\rangle$ as

$$
|\tilde{\phi}\rangle=\{\sqrt{(1-\sqrt{1-\Gamma}) / 2}, 0,0, \sqrt{(1+\sqrt{1-\Gamma}) / 2}\},
$$

and

$$
|\phi\rangle=\{0, \sqrt{1 / 2}, \sqrt{1 / 2}, 0\},
$$

respectively. Then the operator $\hat{M}$ [see Eq. (9)] is represented by

$$
\hat{M}=\frac{1}{2}\left[\begin{array}{cccc}
\mu(1-\sqrt{1-\Gamma}) & 0 & 0 & \mu \sqrt{\Gamma}+1 \\
0 & 1 & 0 & 0 \\
0 & 0 & 1 & 0 \\
\mu \sqrt{\Gamma}+1 & 0 & 0 & \mu(1+\sqrt{1-\Gamma})
\end{array}\right] \text { with } \mu \geqslant 0 \text {. }
$$

The operator $\hat{M}$ has the eigenvector $|e\rangle$ :

$$
|e\rangle \propto\left\{\mu \sqrt{\Gamma}+1,0,0,-\left(\sqrt{\mu^{2}+2 \mu \sqrt{\Gamma}+1}-\mu \sqrt{1-\Gamma}\right)\right\},
$$

which has the negative eigenvalue $\lambda$ :

$$
\lambda=\frac{1}{2}\left(\mu-\sqrt{\mu^{2}+2 \mu \sqrt{\Gamma}+1}\right) .
$$

As $\mu \sqrt{\Gamma}+1>0$ and $-\left(\sqrt{\mu^{2}+2 \mu \sqrt{\Gamma}+1}-\mu \sqrt{1-\Gamma}\right)<0$ hold, we can always determine positive $\mu$ value such that $|\Phi\rangle \propto|e\rangle$ for $|\Phi\rangle=\{s, 0,0, v\}(s>0$ and $v<0)$ by solving

$$
v / s=-\left(\sqrt{\mu^{2}+2 \mu \sqrt{\Gamma}+1}-\mu \sqrt{1-\Gamma}\right) /(\mu \sqrt{\Gamma}+1) .
$$

For example, when $h=1, v / s$ and $\Gamma$ are -0.386 and 0.229 , respectively, and we obtain $\mu=3.147$. Hence, $W$ [see Eq. (20)] and $|\Phi\rangle=\{s, 0,0, v\}$ [see Eq. (21)] meet the optimal LS decomposition.

\section{APPENDIX C}

We mention the derivation of Eq. (20) for $W$. Equations (17) and (18) are written as $X=|B|-E=-W s v$ (C1) and $A D-E^{2}+X^{2}=W\left(A v^{2}+D s^{2}\right)(\mathrm{C} 2)$, respectively. If we introduce a constant $Y \equiv\left[\left(A D-E^{2}\right)+\right.$ 
$\left.X^{2}\right] / X$, we can obtain $-Y s v=A v^{2}+D s^{2}(\mathrm{C} 3)$ by eliminating $W$ from Eq. (C2) using Eq. (C1). We can solve Eq. (C3) with $s^{2}+v^{2}=1$ and obtain $s^{2} / v^{2}=$
$\left\{A^{2} / D^{2}+\left(Y \mp \sqrt{Y^{2}-4 A D}\right)^{2} / 4\right\} /\left\{(A-D)^{2}+Y^{2}\right\}$ (C4). By using Eqs. (C1) and (C3), $W$ is expressed as $W=$ $2 X Y /\left(A v^{2}+D s^{2}\right)$. Finally, we can obtain Eq. (20).
[1] L. Amico, R. Fazio, A. Osterloh, and V. Vedral, Rev. Mod. Phys. 80, 517 (2008).

[2] A. Osterloh, L. Amico, G. Halei, and R. Fazio, Nature 416, 608 (2002).

[3] T. J. Osborne and M. A. Nielsen, Phys. Rev. A 66, 032110 (2002).

[4] B. K. Chakrabarti, A. Dutta, and P. Sen, Quantum Ising Phases and Transitions in Transverse Ising Models (Springer-Verlag, Berlin, 1996).

[5] S. Sachdev, Quantum Phase Transitions (Cambridge University Press, Cambridge, 1999).

[6] W. K. Wootters, Phys. Rev. Lett. 80, 2245 (1998).
[7] M. Lewenstein and A. Sanpera, Phys. Rev. Lett. 80, 2261 (1998).

[8] K. Shimizu and A. Kawaguchi, Phys. Lett. A 355, 176 (2006).

[9] V. Vedral and M. B. Plenio, Phys. Rev. A 57, 1619 (1998).

[10] T. Wellens and M. Kuś, Phys. Rev. A 64, 052302 (2001).

[11] A. Peres, Phys. Rev. Lett. 77, 1413 (1996).

[12] W. Dür, G. Vidal, and J. I. Cirac, Phys. Rev. A 62, 062314 (2000).

[13] K. M. O’Connor and W. K. Wootters, Phys. Rev. A 63, 052302 (2001).

[14] E. Barouch and M. McCoy, Phys. Rev. A 3, 786 (1971).

[15] G. Vidal, J. I. Latorre, E. Rico, and A. Kitaev, Phys. Rev. Lett. 90, 227902 (2003). 